\title{
Therapeutic hypothermia in stroke patients with use of craniocerebral hypothermia helmet: functional outcome and mortality.
}

S. Ustinskaya ${ }^{1}$, I. Sharinova ${ }^{1}$, N. Litvinov ${ }^{1}$, V. Ardashev' ${ }^{2}$ I. Kalenova ${ }^{3}$, A. Kojacary ${ }^{3}$, V. Shmurev ${ }^{3}$, O. Shevelev ${ }^{4}$, M. Petrova ${ }^{4}$ I. Stulin ${ }^{5}$.

${ }^{1}$ FSBI "Presidential hospital №1", Stroke Unit, Moscow, Russia.

${ }^{2} \mathrm{FSBI}$ "Presidential hospital №1", Scientific Director, Moscow, Russia.

3 "Central state medical Academy", Neurology, Moscow, Russia.

${ }^{4}$ RUDN University, Neurology, Moscow, Russia.

${ }^{5}$ A.I. Yevdokimov Moscow State University of Medicine and Dentistry, Neurology, Moscow, Russia.

\section{Background and Aims.}

Craniocerebral hypothermia helmet $(\mathrm{CCH})$ is a non invasive method of hypothermia with preferential the brain cooling. Purpose of the work is to submit functional outcome and mortality in acute stroke patients treated with application of $\mathrm{CCH}$.

\section{Method.}

We have 2 groups of acute stroke patients: $\mathrm{CCH}$ with standard management, and control group standard management without $\mathrm{CCH}$. Inclusion criteria was acute ischemic stroke 72-hours onset. Exclusion criteria was bradycardia.

First group with applications craniocerebral hypothermia helmet consisted of 87 acute stroke patients (mean age $69,7 \pm 12,9$, NIHSS15,4 $\pm 7,1$ points) were treated $\mathrm{CCH}$ and standard protocol. Second group consisted of 20 acute stroke patients (mean age $77,2 \pm 6,2$, NIHSS $12,8 \pm 5,7$ points) were treated standard protocol, without $\mathrm{CCH}$.

We used $\mathrm{CCH}$ for therapeutic hypothermia induction during 24 hours. We calculated Neurological status (NIHSS) before treatment, after 24 hours and after 7 days, modified Rankin Scale points in 14 day and mortality in 21 day after stroke onset.

\section{Results.}

Hypothermia group: NIHSS after 24 hours was10,6 $\pm 7,9$, after 7 days was $8,1 \pm 8,2$. mRS 2,59 $\pm 1,92$ points. In control group: NIHSS after 24 hours was 12,9 $9 \pm 5,9$, after 7 days was 12,5 $\pm 6,8$. mRS 4,25 $\pm 1,62$ points. There was a statistic significant difference of better NIHSS after 7 days $(p=0,028)$, better functional outcome $m R S$ in 14days $(p=0,0005)$ in hypothermia group. Mortality rate $9,1 \%$ in hypothermia group, in control group $25 \%$.

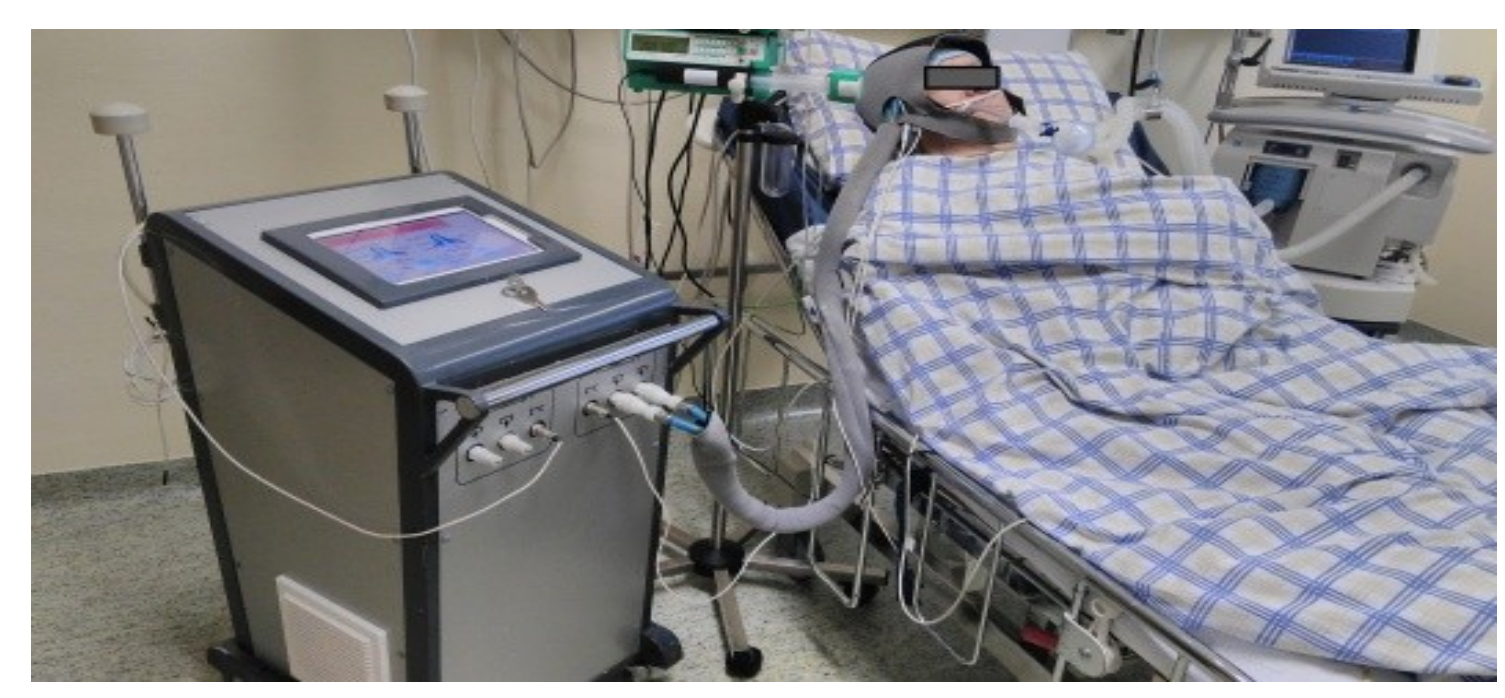

\section{Conclusion.}

We revealed the tendency for better regress of neurological deficit and functional outcome at the patients with $\mathrm{CCH}$. Also, it reduces the rate of disability of patients. $\mathrm{CCH}$ could further improve functional outcome and reduce mortality.

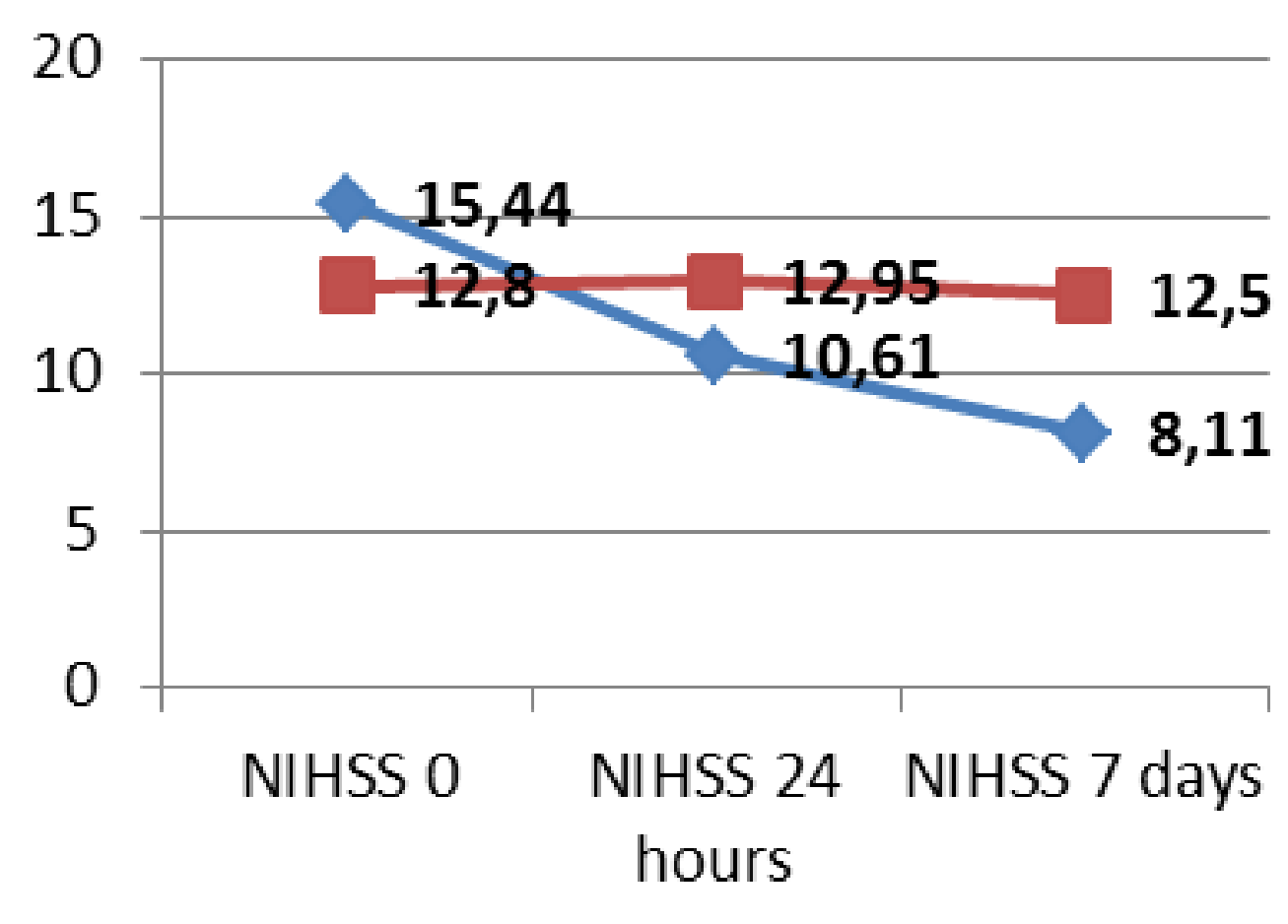

$\longrightarrow$ Hypothermia group - Control group

\section{Mortality}

\begin{tabular}{|c|c|c|c|c|}
\hline Group & Total patients & $\begin{array}{c}\text { Survived patients } \\
\text { after 14 days }\end{array}$ & $\begin{array}{c}\text { Patients died } \\
\text { after 14 days }\end{array}$ & Mortality (\%) \\
\hline Hypothermia & 87 & 79 & 8 & 9,1 \\
\hline Control & 20 & 15 & 5 & 25 \\
\hline
\end{tabular}

\section{Functional outcome (m RS)}

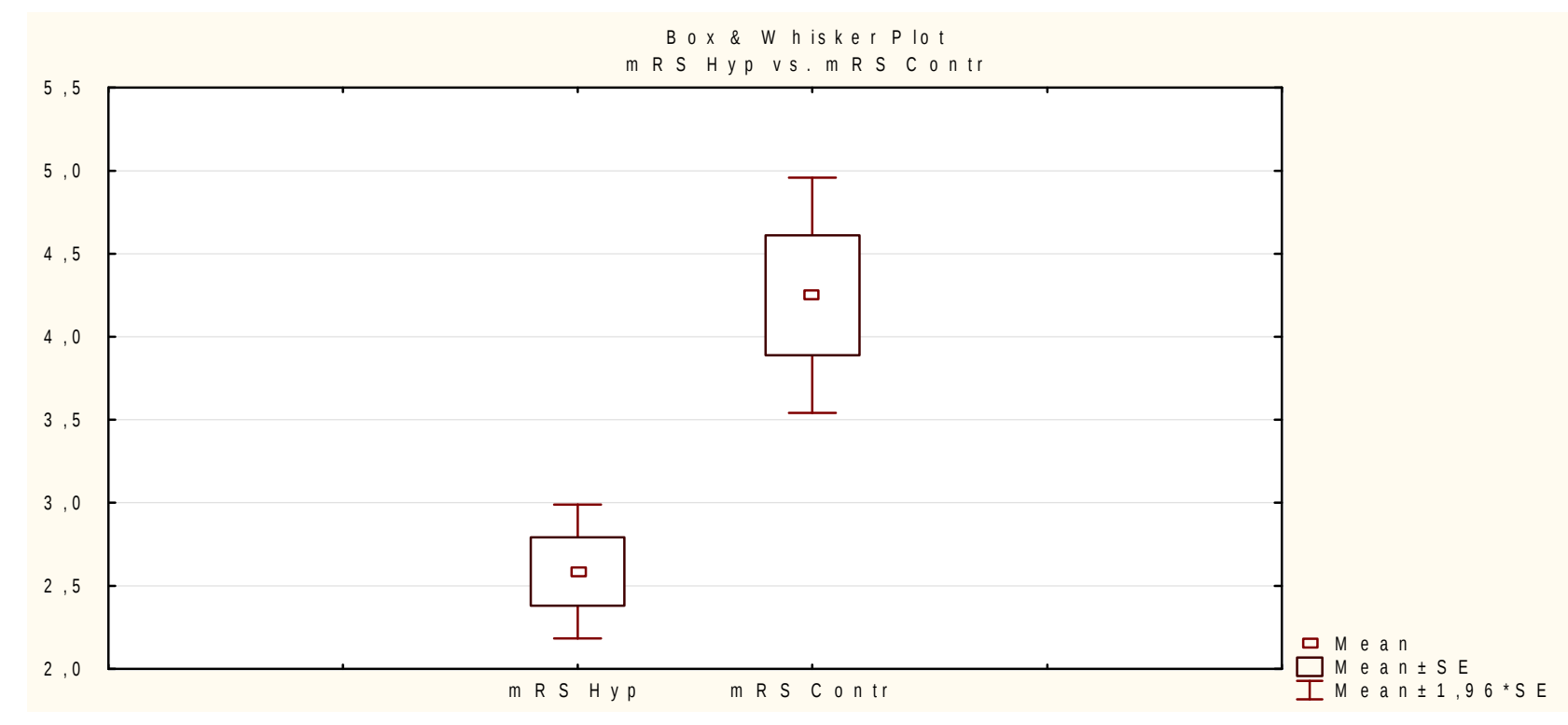

